\section{Red cell distribution width and early mortality in elderly patients with severe sepsis and septic shock}

\author{
Sejin Kim', Kyoungmi Lee', Inbyung Kim', Siyoung Jung', \\ Moon-Jung Kim² \\ Departments of ${ }^{1}$ Emergency Medicine and ${ }^{2}$ Laboratory Medicine, Myongji Hospital, Goyang, Korea
}

Objective To investigate the association of red cell distribution width (RDW) with 30-day mortality in elderly patients with severe sepsis and septic shock.

Methods Patients were recruited from a single tertiary emergency department. Patients with age over 65 years were selected. The main outcome was 30-day mortality. Potential confounders as Acute Physiologic and Chronic Health Evaluation (APACHE) II score and Sequential Organ Failure Assessment (SOFA) score along with initial vital signs were collected. Multivariate Cox proportional hazards analysis was performed to identify independent predictors of 30-day mortality. The discriminative ability of RDW for 30-day mortality was evaluated using receiver operating characteristic curve analysis.

Results Overall, 458 patients were included. Univariate analysis showed that patients' survival was significantly associated with sites of infection, comorbidities, and severity scores. In the multivariate Cox proportional hazard model, the RDW was an independent predictor of 30-day mortality (hazards ratio, 1.10; 95\% confidence interval, 1.04 to $1.17 ; \mathrm{P}<0.001$ ).

Conclusion In this study, initial RDW values were significantly associated with 30-day mortality in older patients hospitalized with severe sepsis and septic shock.

Keywords Sepsis; Mortality; Erythrocyte indices

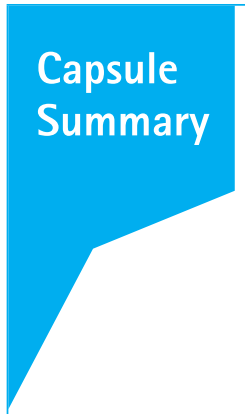

What is already known

The association of red cell distribution width with mortality has been well evaluated in general cardiovascular diseases such as heart failure, acute myocardial infarction, coronary artery disease, pulmonary embolism, cardiac arrest, and stroke.

What is new in the current study

Red cell distribution width could be an outcome predictor in elderly patients with severe sepsis.
eISSN: $2383-4625$

Received: 15 April 2015

Revised: 19 June 2015

Accepted: 20 June 2015

Correspondence to: Kyoungmi Lee Department of Emergency Medicine, Myongji Hospital, 55 Hwasu-ro 14beon-gil, Deogyang-gu, Goyang 10475, Korea

E-mail: gallop94@hanmail.net

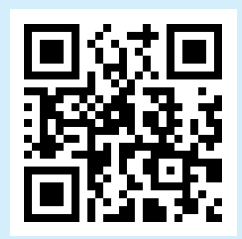

How to cite this article:

Kim S, Lee K, Kim I, Jung S, Kim MJ. Red cell distribution width and early mortality in older adults hospitalized with severe sepsis and septic shock. Clin Exp Emerg Med 2015;2(3):155-161.

This is an Open Access article distributed under the terms of the Creative Commons Attribution Non-Commercial License (http:// creativecommons.org/licenses/by-nc/3.0/). 


\section{INTRODUCTION}

Severe sepsis is defined as sepsis together with sepsis-induced organ dysfunction or tissue hypoperfusion. ${ }^{1}$ Significant advances were accomplished during the last decade in understanding and managing this life-threatening condition. Although mortality from severe sepsis has decreased, the prevalence of severe sepsis and septic shock is increasing. Sepsis is associated with a significantly higher morbidity and mortality in the elderly. ${ }^{2-4}$ Even with current severity scores and biomarkers, predicting outcomes still poses difficulties for clinicians. Identifying patients who will benefit from aggressive treatment and balancing between care and cure for the elderly is very challenging for physicians. ${ }^{5,6}$

Red cell distribution width (RDW) is routinely assessed in emergency settings as part of the complete blood cell count to determine the heterogeneity in the size of circulating erythrocytes. RDW has been reported as a powerful predictor of mortality in various pathological conditions including heart failure, acute myocardial infarction, critical illnesses, and bacterial infections. ${ }^{7-10}$ Recent studies have demonstrated a strong correlation between RDW and mortality in the older population. ${ }^{11-13}$

Although the mechanism of elevated RDW in these patients is yet to be elucidated, an association of inflammation and oxidative stress with elevated RDW has been suggested. ${ }^{14}$ The inflammatory response is important in the pathophysiology of sepsis. Besides, aging is also a powerful prognostic factor of severe sepsis and septic shock. A study of severe sepsis and septic shock indicated a correlation between elevated RDW and early mortality. ${ }^{15}$ Previous studies involving critically ill patients have not focused on older patients with severe sepsis and septic shock. In this study, we aimed to determine whether higher RDW correlates with higher mortality in older adults with severe sepsis and septic shock.

\section{METHODS}

\section{Study population}

We conducted a retrospective study involving elderly patients ( $\geq 65$ years old) with severe sepsis and/or septic shock who visited the emergency department (ED) at an urban, tertiary-care teaching hospital that had an annual ED census of over 50,000 between January 2012 and December 2013. Approximately 16\% of the patients visiting the ED were aged $>65$ years. Patients were identified using Korean Standard Classification of Diseases 6 codes, as well as by an electronic chart review by different program codes (A021, A090, A400-A419, and R572). Severe sepsis was defined as sepsis complicated by organ dysfunction, and sep- tic shock was defined as acute circulatory failure characterized by persistent arterial hypotension that is not explained by other causes. Sepsis-induced hypotension was defined as systolic arterial pressure $<90 \mathrm{mmHg}$ or mean arterial pressure $<60 \mathrm{mmHg}$, or a reduction in systolic arterial pressure of $>40 \mathrm{mmHg}$ from baseline, despite adequate volume resuscitation, and in the absence of other causes of hypotension.' Organ dysfunction was defined using the definition developed by Marshall et al. ${ }^{16} \mathrm{~Pa}-$ tients were managed in accordance with the "Surviving Sepsis Campaign: international guidelines for management of severe sepsis and septic shock 2008."17 Exclusion criteria were as follows: death in the ED, loss to follow-up, and refusal of aggressive treatment.

\section{Laboratory measurements}

Blood samples for the complete blood cell count (including RDW) were collected at the time of admission at the ED. RDW was reported as the coefficient of variation (percentage) of red blood cell volume as measured with an automated analyzer (Beckman Coulter LH780 hematology analyzer; Beckman Coulter, Brea, CA, USA). The normal reference range for RDW in our laboratory is $11.0 \%$ to $15.0 \%$. Other blood tests such as arterial blood gas analyses and serum biochemical analysis were also conducted. Blood culture was performed before initiation of antibiotic therapy, and was defined as positive if aerobic, anaerobic, or fungal organisms were identified.

\section{Data collection}

Clinical data were retrieved from electronic medical records and included information on demographics, comorbidity, foci of infection, blood culture results, length of stay in the ICU, and need for mechanical ventilation. Body temperature, heart rate, respiratory rate, blood pressure, and Glasgow coma scale scores were recorded at the time of triage in the ED. Severity scores, including Acute Physiologic and Chronic Health Evaluation (APACHE) II score and Sequential Organ Failure Assessment (SOFA) score, were also assessed at the time of admission at the ED. The major outcome for this study was 30-day mortality after admission to the ED. A structured telephonic follow-up was performed for patients who were discharged within 30 days of admission.

\section{Statistical analyses}

Patients were grouped into 30-day survivors and non-survivors. Continuous variables were analyzed using Kolmogorov-Smirnov test and Mann-Whitney U-test, and values are expressed as medians and interquartile range. Categorical variables were analyzed using chi square test or Fisher's exact test, and expressed as num- 
Severe sepsis and septic shock patients $\geq 65$ years old

Met KCD 6 criteria in the ED $n=516$

Final selection for analysis $n=417$

Fig. 1. Flow chart of patients enrolled in the study. KCD, Korean Standard Classification of Diseases; ED, emergency department. bers and percentages. A univariate Cox proportional hazards analysis was performed to determine the predictors of mortality in the 30 -day follow-up period. Variables with $\mathrm{P}<0.1$ were included in a multivariate full model with the forward conditional method. The results are expressed as hazards ratio and 95\% confidence interval (Cl). The discriminative ability of RDW for 30-day mortality was evaluated by receiver operating characteristic (ROC) curve analysis. Inter-group differences with associated P-values of $<0.05$ were considered significant. All statistical analyses were performed using SPSS ver. 14.0 (SPSS Inc., Chicago, IL, USA) and Microsoft Excel 2013 (Microsoft, Redmond, WA, USA).

Table 1. Baseline characteristics of the patients

\begin{tabular}{|c|c|c|c|c|}
\hline & Total $(n=458)$ & Survivors $(n=351)$ & Nonsurvivors $(n=107)$ & P-value \\
\hline Age (yr) & 78.0 (73.8-83.0) & $77.0(73.0-82.0)$ & 81.0 (77.0-86.0) & 0.063 \\
\hline Male $(\%)$ & $242(52.8)$ & $183(52.1)$ & $59(55.1)$ & 0.658 \\
\hline \multicolumn{5}{|l|}{ Primary site of infections } \\
\hline Respiratory & $232(50.7)$ & $153(43.6)$ & 79 (73.8) & $<0.001$ \\
\hline Urinary & $104(22.7)$ & $94(26.8)$ & $10(9.3)$ & $<0.001$ \\
\hline Hepatobiliary & 79 (17.2) & 68 (19.4) & $11(10.3)$ & 0.029 \\
\hline Gastrointestinal & $29(6.3)$ & $24(6.8)$ & $5(4.7)$ & 0.503 \\
\hline Miscellaneous & $14(3.1)$ & $12(3.4)$ & $2(1.9)$ & 0.536 \\
\hline \multicolumn{5}{|l|}{ Comorbidities } \\
\hline Diabetes mellitus & $134(29.3)$ & $109(31.1)$ & $25(23.4)$ & 0.145 \\
\hline Hypertension & $282(61.6)$ & $226(64.4)$ & $56(52.3)$ & 0.031 \\
\hline Liver disease & $26(5.7)$ & $20(5.7)$ & $6(5.6)$ & 1.000 \\
\hline COPD & $36(7.9)$ & $30(8.5)$ & $6(5.6)$ & 0.414 \\
\hline Congestive heart failure & $17(3.7)$ & $9(2.6)$ & $8(7.5)$ & 0.035 \\
\hline Chronic renal failure & $28(6.1)$ & $20(5.7)$ & $8(7.5)$ & 0.493 \\
\hline$C A D$ & $47(10.3)$ & $36(10.3)$ & $11(10.3)$ & 1.000 \\
\hline Neoplastic disease & $76(16.6)$ & $54(15.4)$ & $22(20.6)$ & 0.128 \\
\hline \multicolumn{5}{|l|}{ Vital signs } \\
\hline Mean arterial pressure & $83.2(66.7-98.0)$ & $85.0(71.0-100.0)$ & $70.3(55.0-89.0)$ & 0.409 \\
\hline Heart rate & $102.5(84.0-119.0)$ & $100.0(84.0-119.0)$ & $105.0(90.0-119.0)$ & 0.028 \\
\hline Body temperature & $37.0(36.0-38.0)$ & $37.0(36.9-38.0)$ & $37.0(36.0-38.0)$ & $<0.001$ \\
\hline \multicolumn{5}{|l|}{ Laboratory parameters } \\
\hline RDW $(\%)$ & $14.6(13.7-15.9)$ & $14.4(13.6-15.5)$ & $15.4(14.4-17.2)$ & $<0.001$ \\
\hline $\mathrm{pH}$ & 7.41 (7.34-7.47) & $7.42(7.36-7.47)$ & $7.38(7.25-7.44)$ & $<0.001$ \\
\hline $\mathrm{PaO}_{2}(\mathrm{mmHg})$ & $66.5(51.0-74.7)$ & $69.0(54.0-80.0)$ & $58.0(43.0-73.0)$ & $<0.001$ \\
\hline $\mathrm{PaCO}_{2}(\mathrm{mmHg})$ & $32.0(26.8-40.0)$ & $32.0(27.0-40.0)$ & $33.0(25.0-39.0)$ & $<0.001$ \\
\hline Bicarbonate (mmol/L) & $20.6(16.8-24.0)$ & $21.0(17.7-24.3)$ & $18.0(14.1-21.6)$ & 0.871 \\
\hline WBC $\left(10^{3}\right.$ cells $\left./ \mu \mathrm{L}\right)$ & $12.6(8.4-18.0)$ & $12.9(8.8-17.8)$ & $12.0(5.6-19.0)$ & 0.001 \\
\hline Hematocrit $(\%)$ & $35.0(30.0-39.0)$ & $35.0(31.0-39.0)$ & $33.0(28.0-38.0)$ & 0.053 \\
\hline Mean corpuscular volume (fL) & $95.1(91.1-99.0)$ & $94.4(90.7-98.0)$ & $96.8(92.7-101.4)$ & 0.004 \\
\hline Mean corpuscular hemoglobin (pg) & $31.6(30.3-33.0)$ & $31.5(30.3-32.9)$ & $32.2(30.5-33.8)$ & 0.002 \\
\hline Sodium (mmol/L) & $136.5(132.0-140.0)$ & $136.0(132.0-139.0)$ & $138.0(133.0-141.0)$ & $<0.001$ \\
\hline Potassium (mmol/L) & $4.0(3.6-4.5)$ & $4.0(3.6-4.4)$ & $4.2(3.8-4.8)$ & $<0.001$ \\
\hline Blood urea nitrate $(\mathrm{mg} / \mathrm{dL})$ & $26.0(17.2-39.2)$ & $24.7(16.8-35.3)$ & $33.4(20.9-51.2)$ & $<0.001$ \\
\hline Creatinine (mg/dL) & $1.3(1.0-1.9)$ & $1.2(0.9-1.9)$ & $1.5(1.0-2.3)$ & $<0.001$ \\
\hline Cholesterol (mg/dL) & $124.0(97.8-157.0)$ & $130.0(108.0-162.0)$ & $101.0(78.0-132.0)$ & 0.024 \\
\hline Albumin $(\mathrm{g} / \mathrm{dL})$ & $3.2(2.8-3.7)$ & $3.4(2.9-3.7)$ & $2.9(2.5-3.3)$ & $<0.001$ \\
\hline C-reactive protein $(\mathrm{mg} / \mathrm{dL})$ & $11.5(5.4-19.6)$ & $11.3(5.4-19.3)$ & $12.7(5.5-22.7)$ & 0.001 \\
\hline Total bilirubin $(\mathrm{mg} / \mathrm{dL})$ & $0.8(0.5-1.3)$ & $0.8(0.5-1.2)$ & $0.8(0.5-1.4)$ & $<0.001$ \\
\hline
\end{tabular}

Data are presented as median (interquartile range) or number of patients (column \%).

COPD, chronic obstructive pulmonary disease; $C A D$, coronary artery disease; RDW, red cell distribution width; WBC, white blood cell. 


\section{RESULTS}

\section{Baseline demographics and clinical characteristics}

The patient selection flow chart is shown in Fig. 1. A total of 516 patients were included in the study. Of them, 458 patients were included in the final analysis. The median age was 78 years and $242(52.8 \%)$ patients were male. RDW ranged from 12.0 to $32.9 \%$ with a median value of $14.6 \%$ (IOR $13.7 \%$ to $15.9 \%$ ). The demographic characteristics are shown in Table 1 . The overall 30-day mortality of 458 patients was $23 \%$. Patients who died were older and had a higher rate of congestive heart failure. Patients who had pulmonary infections also had a higher mortality rate, while patients with urinary tract infections survived relatively longer. Non-survivors tended to have a lower heart rate. The severity scores of non-survivors at the time of admission to the ED were higher, while the Glasgow coma scale was lower as compared to that of survivors. Among hematologic and laboratory variables, $\mathrm{pH}$ and $\mathrm{PaO}_{2}$, bicarbonate, hematocrit, cholesterol, and albumin levels were significantly higher in survivors than in non-survivors. The mean corpuscular volume (MCV), mean corpuscular hemo-

Table 2. The outcomes of patients

\begin{tabular}{lcccr}
\hline & $\begin{array}{c}\text { Total } \\
(n=458)\end{array}$ & $\begin{array}{c}\text { Survivors } \\
(n=351)\end{array}$ & $\begin{array}{c}\text { Nonsurvivors } \\
(n=107)\end{array}$ & P-value \\
\hline Vasopressor & $286(62.4)$ & $192(54.7)$ & $94(87.9)$ & $<0.001$ \\
Renal replacement therapy & $30(6.6)$ & $14(4.0)$ & $16(15.0)$ & $<0.001$ \\
Mechanical ventilation & $139(30.3)$ & $79(22.5)$ & $60(56.1)$ & $<0.001$ \\
SOFA & $6(4-9)$ & $6(3-8)$ & $9(7-11)$ & 0.003 \\
APACHE II & $16(12-20)$ & $15(12-19)$ & $20(16-26)$ & $<0.001$ \\
ICU LOS (day) & $4(2-10)$ & $4(2-9)$ & $5(2-12)$ & $<0.001$ \\
\hline
\end{tabular}

Data are presented as number of patients (column \%) or median (interquartile range).

SOFA, Sequential Organ Failure Assessment; APACHE, Acute Physiologic and Chronic Health Evaluation; ICU, intensive care unit; LOS, length of stay.

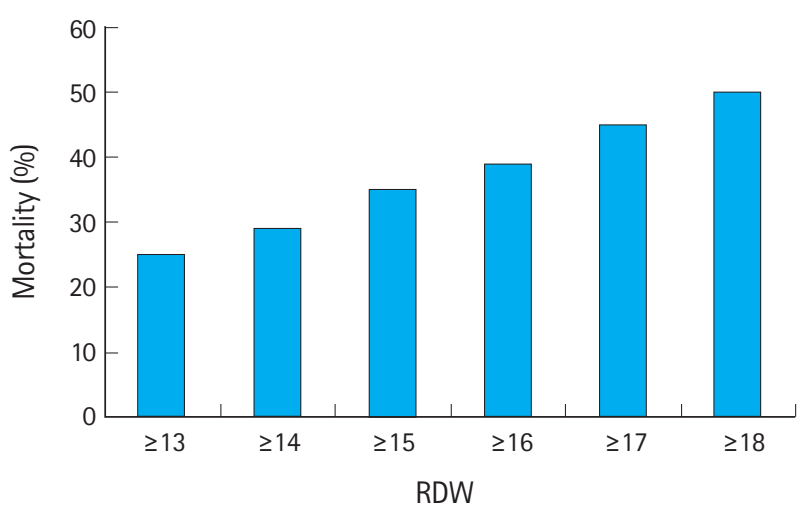

Fig. 2. The 30-day mortality rate according to red cell distribution width (RDW). globin, blood urea nitrogen and potassium levels tended to be higher in non-survivors as compared to the levels in survivors. The median RDW in non-survivors and survivors was $15.4 \%$ and $14.4 \%$, respectively $(\mathrm{P}<0.001)$.

\section{RDW and 30-day mortality}

Non-survivors were treated more aggressively with renal replacement therapy and mechanical ventilator, and had a longer stay in the ICU than survivors (Table 2). Severity scores such as SOFA and APACHE were higher in non-survivors. When dividing RDW into numbers, 30-day mortality increased with increasing RDW values (Fig. 2). Table 3 shows the sensitivity, specificity, and predictive values for 30-day mortality according to RDW values. The higher the RDW value, the lower was its sensitivity and negative predictive value. However, specificity and positive predictive value increased with the increase in percentage of RDW. For each 1\% increase in RDW level as a continuous variable, the 30-day mortality risk increased by $15 \%$ on univariate Cox proportional hazard analysis. In the multivariate Cox proportional hazard model, the RDW was an independent predictor of 30-day mortality after adjustment for other variables (hazards ratio, 1.10; $95 \% \mathrm{Cl}, 1.04$ to

Table 3. Test characteristics of RDW for 30-day mortality

\begin{tabular}{lcccc}
\hline RDW & Sensitivity & Specificity & $\begin{array}{c}\text { Positive } \\
\text { predictive value }\end{array}$ & $\begin{array}{c}\text { Negative } \\
\text { predictive value }\end{array}$ \\
\hline$\geq 13(n=431)$ & 99.1 & 7.1 & 26.7 & 95.7 \\
$\geq 14(n=307)$ & 82.2 & 37.6 & 28.7 & 87.4 \\
$\geq 15(n=182)$ & 58.9 & 66.1 & 34.6 & 84.1 \\
$\geq 16(n=113)$ & 41.1 & 80.3 & 38.9 & 81.7 \\
$\geq 17(n=66)$ & 28.0 & 89.7 & 45.5 & 80.4 \\
$\geq 18(n=40)$ & 18.7 & 94.3 & 50.0 & 79.2 \\
\hline
\end{tabular}

Data are presented as $\%$.

RDW, red cell distribution width.

Table 4. Cox proportional hazard regression analysis for 30-day mortality

\begin{tabular}{lcrr}
\hline & Hazard ratio & 95\% Cl & P-value \\
\hline RDW (per 1\%) & 1.10 & $1.04-1.17$ & 0.001 \\
Age (per 1 year) & 1.07 & $1.04-1.10$ & $<0.001$ \\
SOFA (per 1 point) & 1.11 & $1.02-1.20$ & 0.012 \\
APACHE II (per 1 point) & 1.03 & $1.00-1.07$ & 0.042 \\
Primary site of infection & & & \\
$\quad$ Respiratory & 1.91 & $1.11-3.28$ & 0.020 \\
$\quad$ Urinary & 0.40 & $0.18-0.87$ & 0.022 \\
Bicarbonate (per 1 mmol/L) & 0.93 & $0.89-0.97$ & $<0.001$ \\
MCV (per 1 unit/fL) & 1.03 & $1.01-1.06$ & 0.014 \\
C-reactive protein (per 1 mg/dL) & 1.03 & $1.01-1.04$ & 0.001 \\
Mean arterial pressure (per 1 mmHg) & 0.98 & $0.97-0.99$ & $<0.001$
\end{tabular}

$\mathrm{Cl}$, confidence interval; RDW, red cell distribution width; SOFA, Sequential Organ Failure Assessment; APACHE, Acute Physiologic and Chronic Health Evaluation; $M C V$, mean corpuscular volume. 


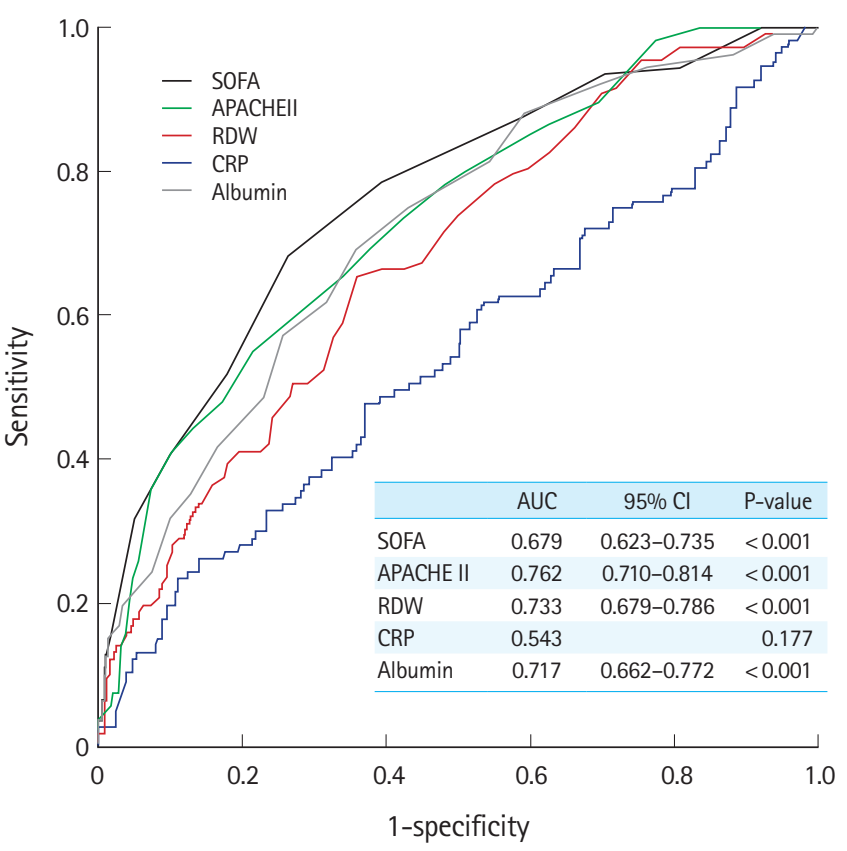

Fig. 3. The receiver operating characteristic curves comparing red cell distribution width (RDW), Sequential Organ Failure Assessment (SOFA) score, Acute Physiologic and Chronic Health Evaluation (APACHE II) score, and albumin and $\mathrm{C}$-reactive protein (CRP) levels to predict 30-day mortality. AUC, area under the ROC curve; $\mathrm{Cl}$, confidence interval.

1.17; $P<0.001)$. Other independent predictors of mortality were age, mean arterial pressure, SOFA score, APACHE II score, pulmonary infection, urinary tract infection, $\mathrm{MCV}$, and $\mathrm{C}$-reactive protein (CRP) level (Table 4).

The utility of RDW along with other variables for predicting 30day mortality was assessed with an ROC curve (Fig. 3). The area under the curve of SOFA score, albumin, APACHE II score, and RDW were $0.679(\mathrm{P}<0.001 ; 95 \% \mathrm{Cl}, 0.619$ to 0.736$), 0.717(\mathrm{P}<0.001$; $95 \% \mathrm{Cl}, 0.662$ to 0.772$), 0.762(\mathrm{P}<0.001 ; 95 \% \mathrm{Cl}, 0.710$ to 0.814$)$, and $0.733(\mathrm{P}<0.001 ; 95 \% \mathrm{Cl}, 0.679$ to 0.786$)$, respectively.

\section{DISCUSSION}

In this study focusing on older patients hospitalized with severe sepsis, RDW strongly and independently predicted 30-day mortality. When adjustments were made for age, mean arterial pressure, MCV, CRP, SOFA score, APACHE II score, and primary site of infections in the multivariate Cox proportional hazards model, for each 1\% increase in RDW level as a continuous variable, 30-day mortality rate increased by $10 \%$. Despite the recent advances in the management of severe sepsis, the effect of the improvement in the context of older population is still obscure. Older adults are more likely to have co-morbidities and are often functionally dependent before admission. It is important to predict the outcomes of severe sepsis in older patients at the time of presentation to the ED. Along with other severity scoring systems, RDW appears to be a good practical tool to evaluate elderly septic patients who require hospitalization.

The association of RDW with mortality has been well documented in patients with common cardiovascular diseases such as heart failure, acute myocardial infarction, coronary artery disease, pulmonary embolism, cardiac arrest, and stroke. ${ }^{8,9,18-25}$ In addition to mortality, RDW has been associated with morbidity in several diseases, e.g., RDW has been associated with the incidence of heart failure and risk of coronary disease. ${ }^{21,26}$ Although the mechanisms linking RDW to adverse patient outcomes are not completely understood, potential pathways include chronic inflammation, malnutrition, and anemia of different etiologies. ${ }^{12,13,18,27,28}$ The prognostic potential of RDW is of particular interest because it is routinely included in the automated complete blood cell count analysis at the ED, and is thus available at no additional cost.

Several studies have found RDW to be of value as a prognostic marker for short-term and long-term mortality in critically ill patients. ${ }^{7,29}$ In a large 10 -year retrospective study, RDW was associated with the risk of disseminated infection and was validated as a strong predictor of 30-day mortality in critically ill patients. ${ }^{7}$ Cohort studies based on the US population have also demonstrated an association of RDW with mortality in community-dwelling patients. ${ }^{12,13,27}$ Other studies have investigated the correlation between RDW and mortality from blood stream infection and gramnegative bacteremia. ${ }^{10,30}$ In these studies, RDW was an independent predictor of 30-day mortality. A recent retrospective study of 566 patients with severe sepsis and septic shock showed a strong correlation of RDW with short-term mortality. ${ }^{15}$ However, none of these studies specifically focused on older patients with sepsis who required ICU admission. Our study involving critically ill older patients demonstrated the value of RDW as an outcome predictor, and that RDW levels may help inform early intervention in elderly patients with severe sepsis.

The underlying mechanism for the association of RDW with mortality in septic patients is still unclear; its elucidation could augment our understanding of the pathophysiology of sepsis, and thereby aid the development of advanced therapeutic modalities for septic patients. Several studies have found RDW to be associated with blood markers of inflammation, such as interlukin-6, CRP, as well as with impaired iron metabolism. ${ }^{18,27}$ Furthermore, oxidative stress has been shown to increase anisocytosis by disrupting erythropoiesis, and to alter red blood cell membrane deformability and red blood cell circulation half-life, ultimately leading to increased RDW. ${ }^{12,27,28}$ In previous studies, adjusting for inflammatory markers such as CRP did not substantially reduce the 
prognostic value of RDW. ${ }^{12,13,27,29}$ Consistent with these findings, we found that the risk associated with RDW was significant even after adjustment for CRP levels. Moreover, RDW alone showed interesting results in the ROC curve analysis, while CRP alone was not a significant predictor of 30-day mortality.

According to Bion, ${ }^{31}$ RDW may also reflect the patients' degree of physiologic reserve, which is one of the three main determinants of clinical outcomes. The physiologic reserve represents the cellular response to acute stress and the resultant tissue hypoxia. Ischemia activates cellular systems that serve to reduce oxygen demand and physiologic processes in order to improve tissue oxygen delivery. As a result, reactive erythropoiesis is stimulated under oxidative stress. Release of large immature red cells, with poor oxygen-binding capacity into the circulation, leads to an increase in the RDW. This whole process could explain the association between RDW and clinical outcomes in acute illness. With advancing age, pro-inflammatory cytokines are thought to inhibit proliferation of erythroid progenitor cells; and to down-regulate erythropoietin receptor expression. ${ }^{28}$ Perturbations in erythropoiesis can lead to more variation in cell sizes and therefore, might increase RDW..$^{13}$ Our study is important in that it explains the mechanism behind RDW representing a prognostic factor in older patients with severe sepsis.

There are several limitations in this study. Firstly, it is a retrospective single-center study based on diagnostic codes and chart review, which is a potential source of bias and errors at the time of data recording at the source. Secondly, since we included only patients who needed ICU care, the findings may not be generalized to all older patients with severe sepsis. Lastly, RDW could be influenced by nutritional status; however, we did not examine variables such as iron, folate, and vitamin B12 levels. Further prospective multi-center studies with larger sample sizes are necessary to further assess the role of RDW as a predictor of mortality.

In this study, initial RDW values were associated with 30-day mortality in older patients hospitalized with severe sepsis and septic shock.

\section{CONFLICT OF INTEREST}

No potential conflict of interest relevant to this article was reported.

\section{REFERENCES}

1. Levy MM, Fink MP, Marshall JC, et al. 2001 SCCM/ESICM/ACCP/ ATS/SIS International Sepsis Definitions Conference. Intensive Care Med 2003;29:530-8.
2. Kaukonen KM, Bailey M, Suzuki S, Pilcher D, Bellomo R. Mortality related to severe sepsis and septic shock among critically ill patients in Australia and New Zealand, 2000-2012. JAMA 2014;311:1308-16.

3. Kumar G, Kumar N, Taneja A, et al. Nationwide trends of severe sepsis in the 21st century (2000-2007). Chest 2011;140: 1223-31.

4. Levy MM, Artigas A, Phillips GS, et al. Outcomes of the Surviving Sepsis Campaign in intensive care units in the USA and Europe: a prospective cohort study. Lancet Infect Dis 2012;12: 919-24.

5. Annane D, Bellissant E, Cavaillon JM. Septic shock. Lancet 2005; 365:63-78.

6. Marshall JC, Reinhart K; International Sepsis Forum. Biomarkers of sepsis. Crit Care Med 2009;37:2290-8.

7. Bazick HS, Chang D, Mahadevappa K, Gibbons FK, Christopher KB. Red cell distribution width and all-cause mortality in critically ill patients. Crit Care Med 2011;39:1913-21.

8. Dabbah S, Hammerman H, Markiewicz W, Aronson D. Relation between red cell distribution width and clinical outcomes after acute myocardial infarction. Am J Cardiol 2010;105:312-7.

9. Felker GM, Allen LA, Pocock SJ, et al. Red cell distribution width as a novel prognostic marker in heart failure: data from the CHARM Program and the Duke Databank. J Am Coll Cardiol 2007;50:40-7.

10. Ku NS, Kim HW, Oh HJ, et al. Red blood cell distribution width is an independent predictor of mortality in patients with gramnegative bacteremia. Shock 2012;38:123-7.

11. Martinez-Velilla N, Ibanez B, Cambra K, Alonso-Renedo J. Red blood cell distribution width, multimorbidity, and the risk of death in hospitalized older patients. Age (Dordr) 2012;34:71723.

12. Patel $K V$, Ferrucci $L$, Ershler WB, Longo DL, Guralnik JM. Red blood cell distribution width and the risk of death in middleaged and older adults. Arch Intern Med 2009;169:515-23.

13. Patel KV, Semba RD, Ferrucci $L$, et al. Red cell distribution width and mortality in older adults: a meta-analysis. J Gerontol A Biol Sci Med Sci 2010;65:258-65.

14. Lippi G, Targher G, Montagnana M, Salvagno GL, Zoppini G, Guidi GC. Relation between red blood cell distribution width and inflammatory biomarkers in a large cohort of unselected outpatients. Arch Pathol Lab Med 2009;133:628-32.

15. Jo $\mathrm{YH}$, Kim K, Lee JH, et al. Red cell distribution width is a prognostic factor in severe sepsis and septic shock. Am J Emerg Med 2013;31:545-8.

16. Marshall JC, Cook DJ, Christou NV, Bernard GR, Sprung CL, Sibbald WJ. Multiple organ dysfunction score: a reliable de- 
scriptor of a complex clinical outcome. Crit Care Med 1995; 23:1638-52.

17. Dellinger RP, Levy MM, Carlet JM, et al. Surviving Sepsis Campaign: international guidelines for management of severe sepsis and septic shock: 2008. Crit Care Med 2008;36:296-327.

18. Allen LA, Felker GM, Mehra MR, et al. Validation and potential mechanisms of red cell distribution width as a prognostic marker in heart failure. J Card Fail 2010;16:230-8.

19. Anderson JL, Ronnow BS, Horne BD, et al. Usefulness of a complete blood count-derived risk score to predict incident mortality in patients with suspected cardiovascular disease. Am J Cardiol 2007;99:169-74.

20. Ani C, Ovbiagele B. Elevated red blood cell distribution width predicts mortality in persons with known stroke. J Neurol Sci 2009;277:103-8.

21. Horne BD, May HT, Kfoury AG, et al. The Intermountain Risk Score (including the red cell distribution width) predicts heart failure and other morbidity endpoints. Eur J Heart Fail 2010; 12:1203-13.

22. Nishizaki Y, Yamagami S, Suzuki H, et al. Red blood cell distribution width as an effective tool for detecting fatal heart failure in super-elderly patients. Intern Med 2012;51:2271-6.

23. Ozsu S, Abul Y, Gunaydin S, Orem A, Ozlu T. Prognostic value of red cell distribution width in patients with pulmonary embolism. Clin Appl Thromb Hemost 2014;20:365-70.
24. Zalawadiya SK, Veeranna V, Niraj A, Pradhan J, Afonso L. Red cell distribution width and risk of coronary heart disease events. Am J Cardiol 2010;106:988-93.

25. Kim J, Kim K, Lee JH, et al. Red blood cell distribution width as an independent predictor of all-cause mortality in out of hospital cardiac arrest. Resuscitation 2012;83:1248-52.

26. Tonelli M, Sacks F, Arnold M, et al. Relation between red blood cell distribution width and cardiovascular event rate in people with coronary disease. Circulation 2008;117:163-8.

27. Perlstein TS, Weuve J, Pfeffer MA, Beckman JA. Red blood cell distribution width and mortality risk in a community-based prospective cohort. Arch Intern Med 2009;169:588-94.

28. Weiss G, Goodnough LT. Anemia of chronic disease. N Engl J Med 2005;352:1011-23.

29. Wang F, Pan W, Pan S, Ge J, Wang S, Chen M. Red cell distribution width as a novel predictor of mortality in ICU patients. Ann Med 2011;43:40-6.

30. Yeh CF, Chen KF, Ye JJ, Huang CT. Derivation of a clinical prediction rule for bloodstream infection mortality of patients visiting the emergency department based on predisposition, infection, response, and organ dysfunction concept. J Microbiol Immunol Infect 2014;47:469-77.

31. Bion JF. Susceptibility to critical illness: reserve, response and therapy. Intensive Care Med 2000;26 Suppl 1:S57-63. 